\title{
Use of bacteriophages in the treatment of colistin-only-sensitive Pseudomonas aeruginosa septicaemia in a patient with acute kidney injury - a case report
}

\author{
Serge Jennes ${ }^{1}$, Maia Merabishvili², Patrick Soentjens ${ }^{3}$, Kim Win Pang $^{3}$, Thomas Rose ${ }^{1}$, Elkana Keersebilck ${ }^{1}$ \\ Olivier Soete', Pierre-Michel François', Simona Teodorescu', Gunther Verween², Gilbert Verbeken², \\ Daniel De Vos ${ }^{2}$ and Jean-Paul Pirnay ${ }^{2^{*}}$ (1)
}

Keywords: Pseudomonas aeruginosa, Antibiotic resistance, Colistin, Bacteraemia, Acute kidney injury, Intravenous, Bacteriophage therapy

Sepsis from Pseudomonas aeruginosa bacteraemia may be fatal, especially when no appropriate therapy can be given.

In June 2016, a 61-year-old man was hospitalised for Enterobacter cloacae peritonitis and severe abdominal sepsis with disseminated intravascular coagulation, secondary to a diaphragmatic hernia with bowel strangulation. The patient had a prolonged hospital course complicated by gangrene of the peripheral extremities, resulting in the amputation of the lower limbs and the development of large necrotic pressure sores (Fig. 1).

Three months after admission, the patient was transferred to the Queen Astrid military hospital for surgical management of the pressure sores. Wound cultures on admission revealed colonisation with, amongst others, multidrug-resistant $P$. aeruginosa. One month after admission, the patient developed septicaemia with colistin-only-sensitive $P$. aeruginosa. Intravenous colistin therapy was started.

Ten days later, the patient developed acute kidney injury with rising serum creatinine and urea levels (Fig. 2), presumably sepsis- and drug-induced. Antibiotic therapy was discontinued to prevent further kidney damage. Unfortunately, on 12 November, $P$. aeruginosa septicaemia re-emerged with rapid heart rate, low blood pressure,

\footnotetext{
* Correspondence: jean-paul.pirnay@mil.be

${ }^{2}$ Laboratory for molecular and cellular technology, Queen Astrid military

hospital, Brussels, Belgium

Full list of author information is available at the end of the article
}

fever and high C-reactive protein (CRP) levels. On 14 November, the patient went into a coma with 9 scores (E2V2M5) on the Glasgow Coma Scale. Because of the risk of colistin nephrotoxicity and the family's will to avoid intensive therapy interventions such as hemofiltration, bacteriophage therapy was initiated under the umbrella of Article 37 (Unproven Interventions in Clinical Practice) of the Declaration of Helsinki [1]. Fifty microlitres of purified bacteriophage cocktail BFC1 [2], containing two bacteriophages that showed in vitro activity against the patient's $P$. aeruginosa

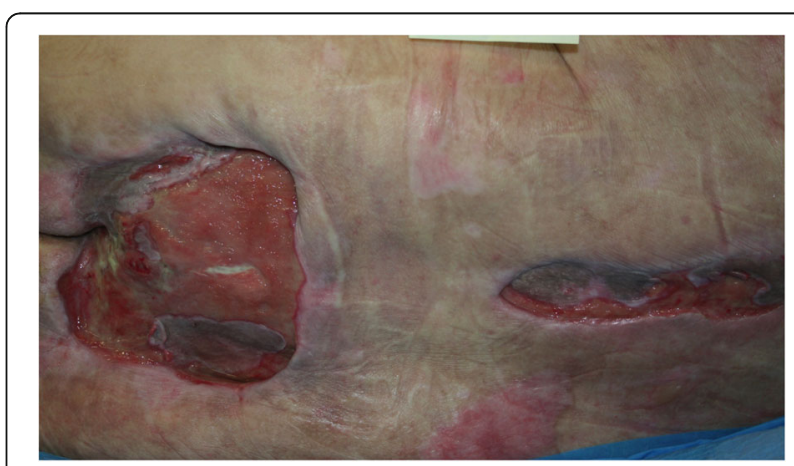

Fig. 1 Large pressure sores in the sacral and spinal back areas. Situation on 17 November 2016 


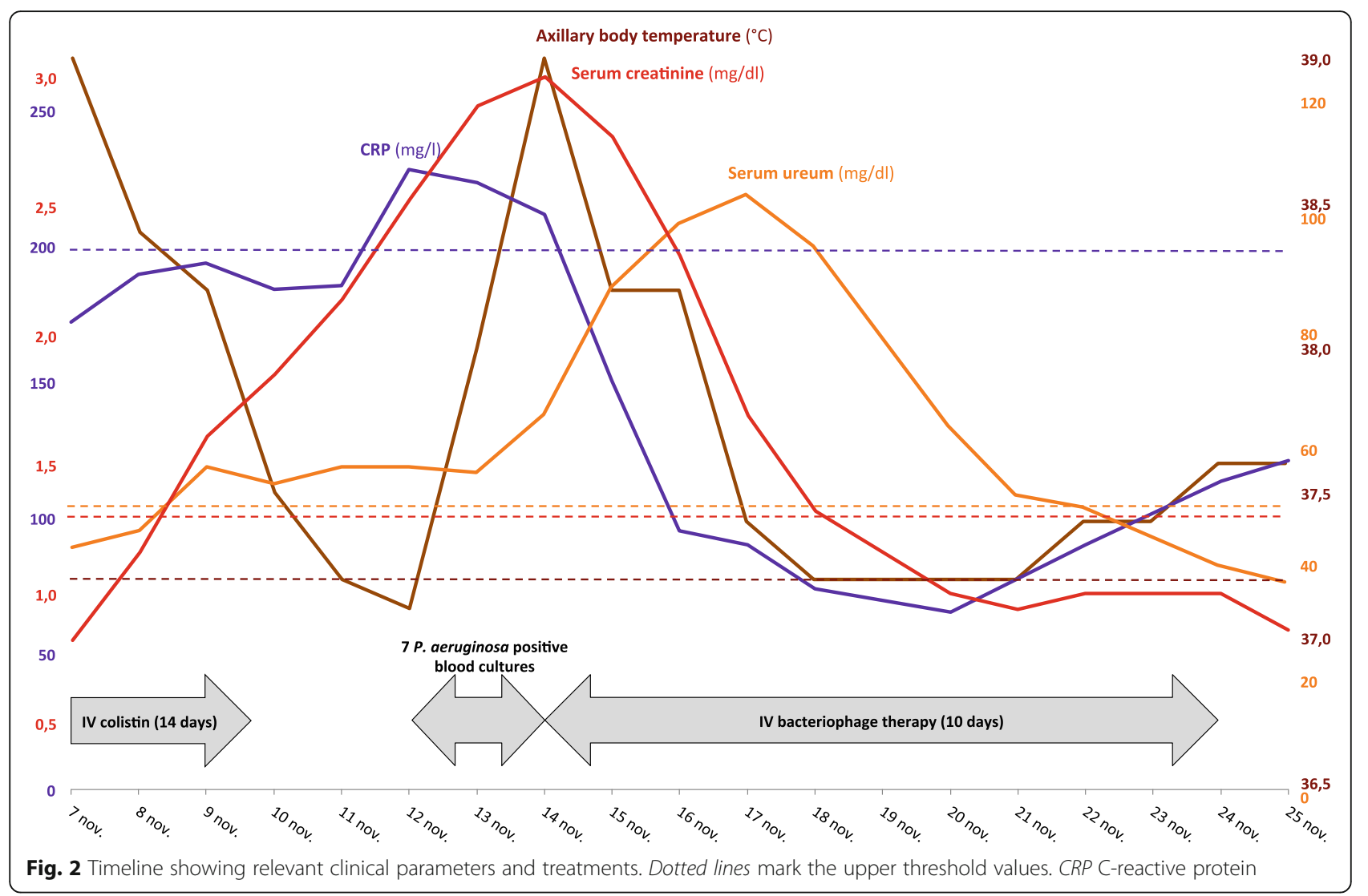

isolates, were administered as a 6-h intravenous infusion for 10 days. Wounds were irrigated with $50 \mathrm{ml}$ BFC1 every $8 \mathrm{~h}$ for 10 days.

Immediately, blood cultures turned negative, CRP levels dropped and the fever disappeared (Fig. 2). Kidney function recovered after a few days (Fig. 2). Hemofiltration was avoided and no unexpected adverse events, clinical abnormalities or changes in laboratory test results that could be related to the application of bacteriophages were observed. The pressure sores remained infected with several bacterial species, including $P$. aeruginosa, causing multiple episodes of sepsis, which were treated using empirical antibiotic therapy. Unfortunately, the patient died 4 months after bacteriophage therapy of sudden in-hospital refractory cardiac arrest due to blood culture-confirmed Klebsiella pneumoniae sepsis. In vitro susceptibility testing revealed that the $K$. pneumoniae strain was sensitive to the antibiotics the patient was on.

Bacteriophages are increasingly put forward as safe alternatives or additions to antibiotic therapy. Historical reports show that they were efficaciously used via the intravenous route, especially in typhoid fever and Staphylococcus aureus bacteremia [3], but this is-as far as we know-the first contemporary report of intravenous bacteriophage monotherapy against $P$. aeruginosa septicaemia in humans.
Abbreviations

CRP: C-reactive protein; IV: intravenous

Acknowledgements

Not applicable.

\section{Funding}

MM received funding (grant number HFM 15-5) from the Royal Higher Institute for Defence.

\section{Availability of data and materials}

The datasets analysed during this case report are available from the corresponding author on reasonable request.

\section{Authors' contributions}

SJ, PS, TR, EK, OS, PMF and ST were involved in patient management. SJ, KWP, PS, DDV and JPP were involved in data analysis. MM, GuV, GiV, DDV and JPP were involved in bacteriophage research and development, production and QA/QC/RA. SJ and JPP drafted the initial report. All authors read and approved the final manuscript.

\section{Competing interests}

The authors declare that they have no competing interests.

\section{Consent for publication}

Written consent to publish was received from the patient's family as part of the terms and conditions of Article 37 of the declaration of Helsinki, which states "in all cases, new information must be recorded and, where appropriate, made publicly available".

\section{Ethics approval and consent to participate}

In concordance with Article 37 of the Declaration of Helsinki, bacteriophage therapy was initiated upon expert advice and with informed consent from the patient's family. 
Data collection required no contact with the patients and no clinical samples other than those relevant for the treatment were collected from patients. Noninterventional retrospective data analysis is not subjected to the Belgian law concerning experiments on the human person (May 7, 2004) and ethics committee approval is not required.

\section{Publisher's Note}

Springer Nature remains neutral with regard to jurisdictional claims in published maps and institutional affiliations.

\section{Author details}

${ }^{1}$ Burn wound center, Queen Astrid military hospital, Brussels, Belgium. ${ }^{2}$ Laboratory for molecular and cellular technology, Queen Astrid military hospital, Brussels, Belgium. ${ }^{3}$ Phage therapy center, Queen Astrid military hospital, Brussels, Belgium.

Published online: 04 June 2017

\section{References}

1. World Medical Association. Declaration of Helsinki: Ethical Principles for Medical Research Involving Human Subjects. JAMA. 2013;310:2191-4.

2. Merabishvili M, Pirnay JP, Verbeken G, Chanishvili N, Tediashvili M, Lashkhi N, et al. Quality-controlled small-scale production of a well-defined bacteriophage cocktail for use in human clinical trials. PLoS One. 2009:4:e4944.

3. Speck P, Smithyman A. Safety and efficacy of phage therapy via the intravenous route. FEMS Microbiol Lett. 2016:363:fnv242. 